\title{
DNA Content of Individual Mitochondrial Nucleoids Varies Depending on the Culture Conditions of the Yeast Saccharomyces cerevisiae
}

\author{
Isamu Miyakawa ${ }^{1, *}$, Masahiro Miyamoto ${ }^{1}$, Tsuneyoshi Kuroiwa ${ }^{2}$ \\ and Nobundo Sando ${ }^{1,3}$ \\ ${ }^{1}$ Department of Physics, Informatics and Biology, Faculty of Science, Yamaguchi University (formerly named \\ the Biological Institute, Faculty of Science, Yamaguchi University), Yamaguchi 753-8512, Japan \\ ${ }^{2}$ Department of Life Sciences, College of Science, Rikkyo University, Tokyo 171-8501, Japan \\ ${ }^{3}$ Present address: 4-39-13 Goko, Matsudo 270-2213, Japan
}

Received February 12, 2004; accepted March 9, 2004

\begin{abstract}
Summary The DNA content of individual mitochondrial nucleoids (mt-nucleoids) in the stationary-phase cells of the yeast Saccharomyces cerevisiae was quantitatively measured by a combination of DAPI-staining and use of a video-intensified microscope photon counting system (VIMPCS). The mt-nucleoids in the cells which were grown aerobically in AN medium contained, on average, 1.5 mitochondrial DNA (mtDNA) molecules per mt-nucleoid, and the mt-nucleoids that were isolated from these cells contained 1.1 mtDNA molecules per mt-nucleoid. In contrast, the giant mt-nucleoids that appeared in the cells grown anaerobically in the same medium contained, on average, 20.3 mtDNA molecules per mt-nucleoid, and the giant mt-nucleoids that were isolated from these cells contained, on average, $13.1 \mathrm{mtDNA}$ molecules per mt-nucleoid. These results, together with the previous data, demonstrated that the DNA content of individual mt-nucleoids and the number of mtnucleoids in a cell were distinctly changed by the culture conditions of the yeast cells, whereas the total mtDNA content per cell did not vary significantly when the culture conditions were changed.
\end{abstract}

Key words Yeast, Saccharomyces cerevisiae, Mitochondria, Mitochondrial nucleoids, Anaerobic culture.

Apparently, mitochondrial DNA (mtDNA) does not exist in a plain form, but, rather, as DNAprotein complexes (mt-nucleoids) within mitochondria (Kuroiwa 1982, Kawano et al. 1995). Analyses of the mt-nucleoid proteins of the yeast Saccharomyces cerevisiae have shown that a major DNA-binding protein, Abf2p, and several other mitochondrial proteins play important roles in mtnucleoid organization (Miyakawa et al. 1987, 1995, 1996, 2000, Miyakawa and Sato 2001, Newman et al. 1996, Sato et al. 2002). The dynamics of mt-nucleoids during the life cycle of the yeast was also extensively examined by fluorescence microscopy (Miyakawa et al. 1984, 1994).

In $S$. cerevisiae, there is a contradiction called a "ploidy paradox" between the large physical number of mtDNA molecules (50-100) per cell, and the small number (1-5) of heritable units, which were estimated from genetic studies of mitochondrial genomes (Piskur 1994). Although the reason for this contradiction is still not well understood, it may partly result from the fact that several mtDNA molecules are packaged together in an mt-nucleoid, which is transmitted as a heritable unit. Therefore, it is important to find out how many molecules of mtDNA are contained in each mt-nucleoid in yeast cells, and how the DNA content per mt-nucleoid and the number of mt-nucleoids per cell are regulated.

In a previous study, we successfully isolated mt-nucleoids from stationary-phase cells of $S$. cerevisiae, which were grown aerobically in a modified Burkholder's medium, and showed that individual mt-nucleoids in spheroplasts and in the mt-nucleoid fractions contained, on average, 3.9

\footnotetext{
* Corresponding author, e-mail: miyakawa@yamaguchi-u.ac.jp
} 
and 3.1 mtDNA molecules, respectively (Miyakawa et al. 1987). Shiiba et al. (1997) also showed that the anaerobic culture of yeast cells introduced a marked aggregation of mt-nucleoids and a reduction in the number of mt-nucleoids per cell, indicating that the size and number of mt-nucleoids showed striking changes depending on the culture conditions. However, the number of mtDNA molecules per mt-nucleoid has not been accurately quantified, except in our previous report (Miyakawa et al. 1987). In this report, we determined the DNA content of individual mt-nucleoids in yeast cells that were grown either anaerobically or aerobically by a combination of DAPI-staining and by use of a video-intensified microscope photon counting system (VIMPCS).

\section{Materials and methods}

\section{Strains and culture}

The diploid strain G2-2 of Saccharomyces cerevisiae was used (Miyakawa et al. 1984). The anaerobic culture was performed as previously described (Shiiba et al. 1997). The anaerobic (AN) medium used was basically the same medium as that used by Tustanoff and Bartley (1964), and consisted of $10.0 \mathrm{~g}$ yeast extract, $5.0 \mathrm{~g}$ casamino acids (Difco, vitamin-free), $9.0 \mathrm{~g} \mathrm{KH}_{2} \mathrm{PO}_{4}, 6.0 \mathrm{~g}$ $\left(\mathrm{NH}_{4}\right)_{2} \mathrm{SO}_{4}, 0.5 \mathrm{~g} \mathrm{MgSO}_{4} 7 \mathrm{H}_{2} \mathrm{O}, 0.3 \mathrm{~g} \mathrm{CaCl}_{2}, 5.83 \mathrm{~g}$ sodium lactate $(60 \% \mathrm{w} / \mathrm{w}), 0.02 \mathrm{~g}$ ergosterol, $5.0 \mathrm{ml}$ Tween $80,0.6 \mathrm{mg}$ wheat germ oil and $50 \mathrm{~g}$ glucose per liter ( $\mathrm{pH} 5.3$ ).

The aerobic cultures were performed in $100-\mathrm{ml}$ of AN medium or a modified Burkholder's medium (BT medium) supplemented with 3.6\% (v/v) tomato extract (Sando and Miyake 1971), under reciprocal shaking at $30^{\circ} \mathrm{C}$ up to the stationary phase.

\section{Isolation of mt-nucleoids}

The cells were converted to spheroplasts by treatment with Zymolyase 100T (Seikagaku Kogyo Co., Ltd., Tokyo, Japan) (Miyakawa et al. 1987). To avoid morphological changes of the mtnucleoids during spheroplast formation of the anaerobically-grown cells, $\mathrm{N}_{2}$ gas was adequately bubbled in the solutions throughout spheroplast formation and the isolation of the mitochondria, in order to minimize the exposure to $\mathrm{O}_{2}$ (Shiiba et al. 1997).

The isolation of giant mt-nucleoids from the anaerobically-grown cells was performed as previously described (Shiiba et al. 1997). The mt-nucleoids from aerobically-grown cells in BT medium were isolated according to the conventional methods described previously (Miyakawa et al. 1987). Mt-nucleoids from cells grown aerobically in AN medium were isolated using a slightly modified method, due to their smaller size. In this case, the mt-nucleoids were fractionated on a $20 \%, 40 \%$, and $60 \%(\mathrm{w} / \mathrm{v})$ discontinuous sucrose gradient at $131,000 \mathrm{~g}$ for $1 \mathrm{~h}$, instead of $46,000 \mathrm{~g}$ for $1 \mathrm{~h}$, and the mt-nucleoids at the boundary layer between $40 \%$ and $60 \%$ were collected and pooled.

\section{Fluorescence microscopy}

An epifluorescence microscope (BHS-RFK; Olympus Optical Co., Ltd., Tokyo, Japan) equipped with appropriate objectives (Dplan Apo 100UVPL and 100UV; Olympus) was used. The spheroplasts were fixed with $1 \%$ glutaraldehyde at room temperature for $1 \mathrm{~h}$. The specimens were mixed with an equal volume of a solution consisting of 4',6-diamidino-2-phenylindole (DAPI) $(2 \mu \mathrm{g} / \mathrm{ml})$ dissolved in NS buffer on a glass slide, and squashed (Miyakawa et al. 1984).

The DNA content of the mt-nucleoids was determined by measuring the fluorescence intensity of each mt-nucleoid stained with DAPI $(1 \mu \mathrm{g} / \mathrm{ml})$, using a video-intensified microscope photon counting system (VIMPCS) (Hamamatsu Photonics Ltd., Hamamatsu, Japan), which was connected to an epifluorescence microscope. The fluorescence intensity of the T4 phage after the DAPI-staining was used as a standard to express the relative fluorescence intensity. The number (X) of mtDNA molecules contained in an mt-nucleoid of various samples was calculated using the value of the 
mean photon number $(77,000$ counts per $2 \mathrm{~s}), \mathrm{G}+\mathrm{C}$ content $30 \%$, DNA content $110 \times 10^{6}$ of $\mathrm{T} 4$ phage as standard, and the mean photon number (A) of mt-nucleoids, molecular weight $50 \times 10^{6}$, $\mathrm{G}+\mathrm{C}$ content $18 \%$ of $S$. cerevisiae mtDNA, and the equation $\mathrm{X}=0.223 \mathrm{~A}$ (Miyakawa et al. 1987). Photographs were taken using Fuji Neopan 400 (ASA 400) film with an exposure time of 16 sec. The number of mt-nucleoids in each strongly squashed spheroplast was counted on the photomicrographs, which were enlarged from the film negatives.

\section{Results}

Figure 1 shows photomicrographs of the squashed spheroplasts of yeast cells grown under three different conditions (Fig. 1A-C), and of the mt-nucleoids isolated from cells grown on each culture (Fig. 1D-F). The spheroplasts of the cells that were grown aerobically in BT medium had many discrete spots of mt-nucleoids (Fig. 1A) (Miyakawa et al. 1987). The mean number \pm SD of the mt-nucleoids was $63.4 \pm 21.6$ per cell (Table 1). Similar-sized mt-nucleoids were isolated from those spheroplasts (Fig. 1D). However, the spheroplasts of cells that were grown aerobically in AN medium (Fig. 1B) had a larger number of mt-nucleoids than those of cells that were grown in BT medium (Fig. 1A). The mean number \pm SD of mt-nucleoids was $141.4 \pm 52.1$ per cell (Table 1$)$. In contrast, the spheroplasts of cells that were grown anaerobically in AN medium had far fewer mtnucleoids, and these were distinctly larger in size than those that appeared in the aerobically-grown cells (Fig. 1C). The mean number \pm SD of mt-nucleoids per cell was $7.6 \pm 3.7$ (Table 1). The mt-nucleoids that were isolated from those cells also contained large aggregated forms of the mt-nucleoids (Fig. 1F).
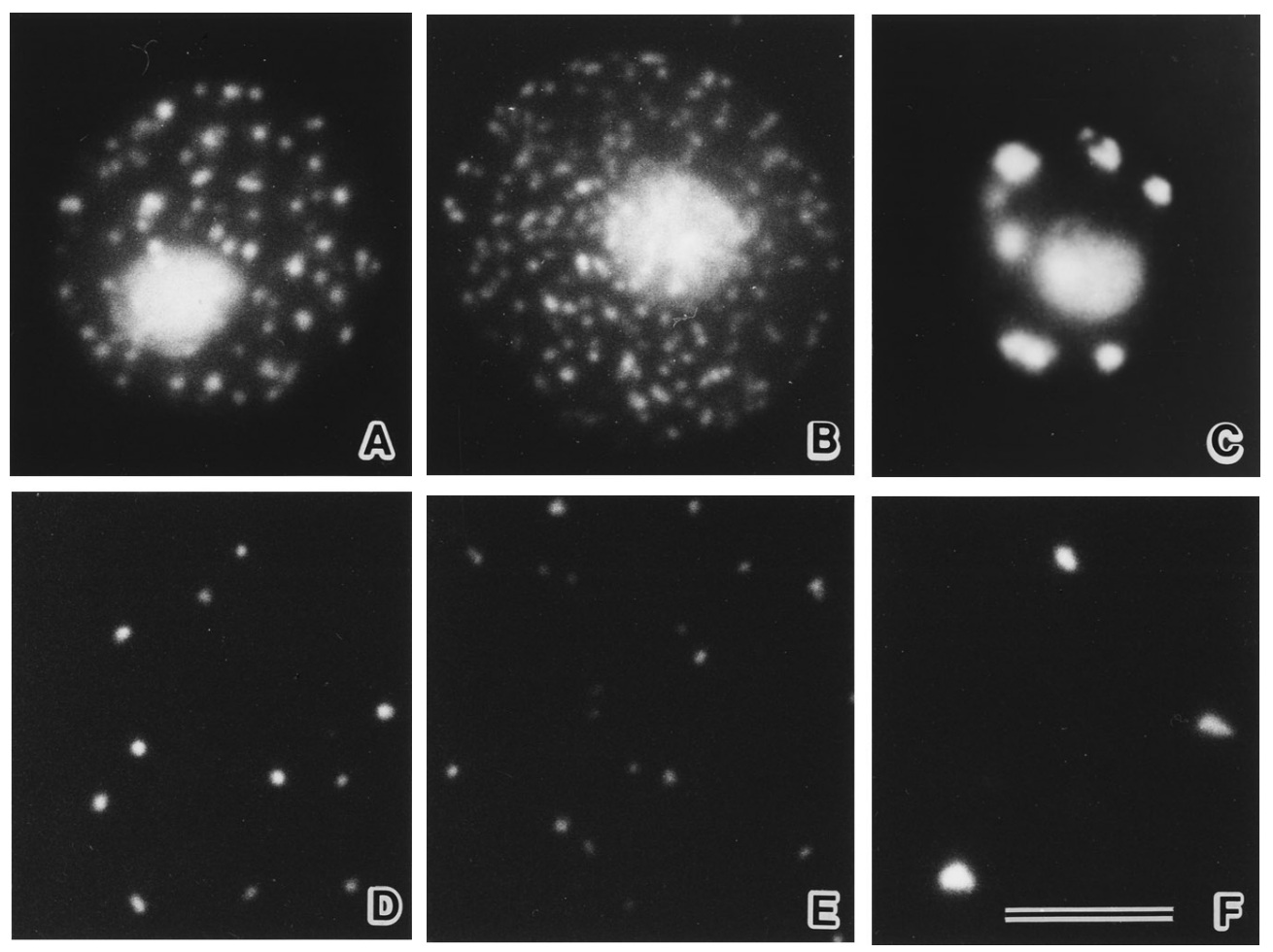

Fig. 1. DAPI-fluorescence photomicrographs of spheroplasts at the stationary phase and the isolated mt-nucleoids. A) A cell grown aerobically in BT medium, B) A cell grown aerobically in AN medium, C) A cell grown anaerobically in AN medium. D), E) and F) are the mt-nucleoids isolated from cells A, $\mathrm{B}$ and $\mathrm{C}$, respectively. Bar represents $5 \mu \mathrm{m}$. 
Table 1. The number of mtDNA molecules per mt-nucleoid and the number of mt-nucleoids per cell in S. cerevisiae grown under three different conditions

\begin{tabular}{lccc}
\hline \hline \multicolumn{1}{c}{ Culture conditions } & $\begin{array}{c}\text { Number of mtDNA } \\
\text { per nucleoid } \\
\text { mean } \pm \text { SD }\end{array}$ & $\begin{array}{c}\text { Number of nucleoids } \\
\text { per cell } \\
\text { mean } \pm \text { SD }\end{array}$ & $\begin{array}{c}\text { Number of mtDNA } \\
\text { per cell }\end{array}$ \\
\hline Aerobic culture in BT medium & $3.9 \pm 1.0^{*}$ & $63.4 \pm 21.6$ & 247.3 \\
Aerobic culture in AN medium & $1.5 \pm 0.5$ & $141.4 \pm 52.1$ & 212.1 \\
Anaerobic culture in AN medium & $20.3 \pm 15.1$ & $7.6 \pm 3.7$ & 154.3 \\
\hline
\end{tabular}

* Cited from previous data (Miyakawa et al. 1987).

(A)

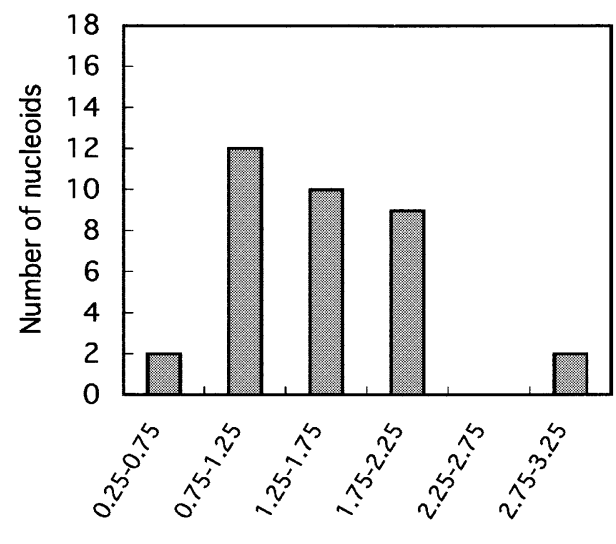

mtDNA molecules/nucleoid
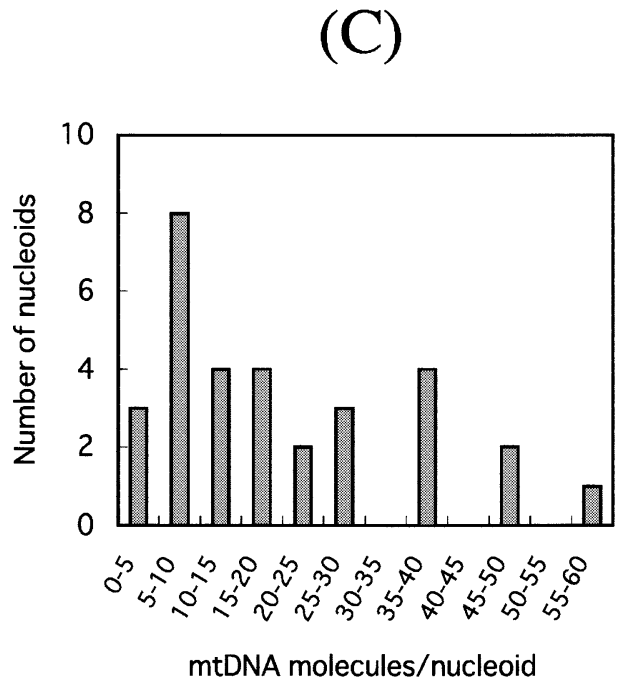

(B)

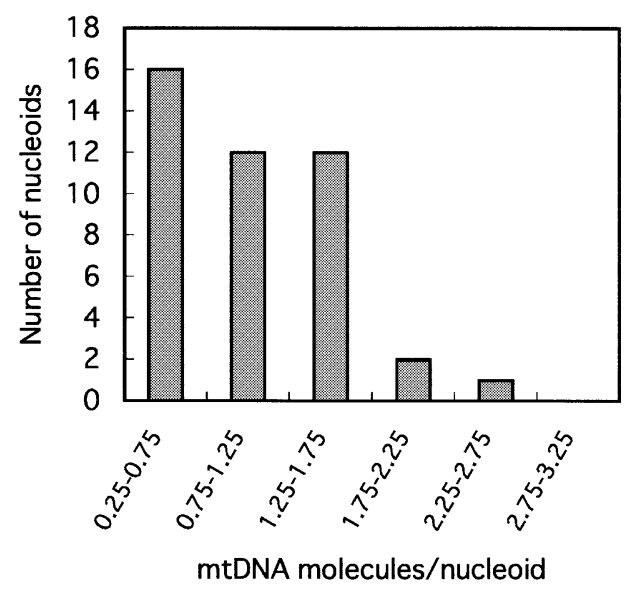

(D)

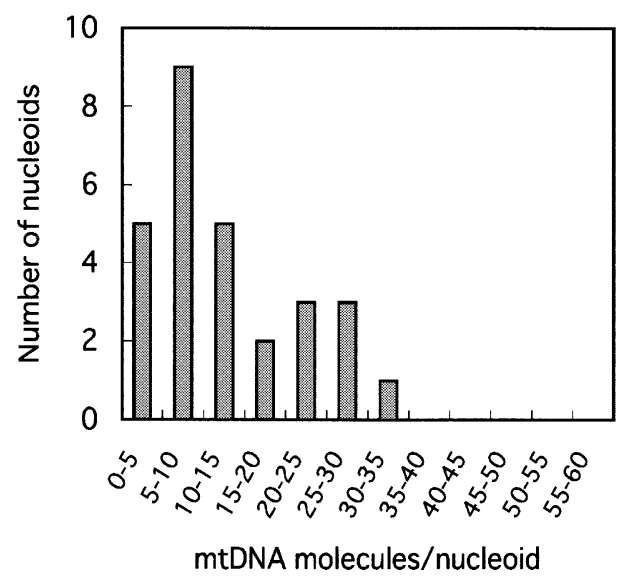

Fig. 2. The number of mtDNA molecules in mt-nucleoids examined by a video-intensified microscope photon counting system. A) mt-nucleoids in spheroplasts grown aerobically in AN medium, B) mt-nucleoids isolated from spheroplasts of cells grown aerobically in AN medium, C) mt-nucleoids in spheroplasts grown anaerobically in AN medium, D) mt-nucleoids isolated from spheroplasts of cells grown anaerobically in AN medium. 
The DNA content of individual mt-nucleoids was measured in the spheroplasts and in the isolated mt-nucleoids by VIMPCS. The histograms of the DNA content per mt-nucleoid are shown in Fig. 2. In the spheroplasts of the cells that were grown aerobically in AN medium, almost all of the mt-nucleoids had mtDNA molecules of between 1 and 2 , and the mean number \pm SD was $1.5 \pm 0.5$ per mt-nucleoid (Fig. 2A, Table 1). The mt-nucleoids that were isolated from those spheroplasts contained a somewhat smaller number of mtDNA, and the mean number \pm SD was $1.1 \pm 0.5$ per mtnucleoid (Fig. 2B). In contrast, the mt-nucleoids in the spheroplasts of the cells that were grown anaerobically in AN medium had a distinctly larger number of mtDNA, though the DNA content per mt-nucleoid significantly varied among the mt-nucleoids. The mean number \pm SD per mt-nucleoid reached 20.3 $\pm 15.1 \mathrm{mtDNA}$ molecules (Fig. 2C, Table 1). The isolated mt-nucleoids contained a somewhat smaller number of mtDNA molecules, and the mean number \pm SD was $13.1 \pm 8.8$ per mt-nucleoid (Fig. 2D).

The number of mtDNA molecules per cell can be estimated from the number of mt-nucleoids per cell and the number of mtDNA molecules per mt-nucleoid. The number of mtDNA molecules per cell was calculated as 247.3 in the cells grown aerobically in BT medium, 212.1 in the cells grown aerobically in AN medium, and 154.3 in the cells grown anaerobically in AN medium, respectively (Table 1).

\section{Discussion}

A number of studies have been performed to determine the mtDNA content of various strains of yeast cells, including wild-type cells ( $\mathrm{rho}^{+}$cells) and respiratory-deficient cells ( $\mathrm{rho}^{-}$cells) grown under various culture conditions (anaerobic, aerobic, glucose-repressed and de-repressed conditions). These studies were based on biochemical analyses of mtDNA after the extraction of DNA from cells. So far, it is generally accepted that mtDNA represents an average of about $15 \%$ (5-25\% range) of the total DNA of a haploid yeast cell (Williamson et al. 1977, Dujon 1981).

However, these biochemical analyses have not presented any information about how many molecules of mtDNA are compactly packaged into individual mt-nucleoids. In order to answer this question, the DNA content of individual mt-nucleoids must be directly determined. In the present study, quantification of the mtDNA content per mt-nucleoid was performed by DAPI-staining and by using VIMPCS, and with these methods, distinct changes of DNA content per mt-nucleoid in yeast cells were demonstrated. It was shown that in cells that were grown aerobically in AN medium, only low contents of mtDNA, equivalent to 1 or 2 molecules, were compactly folded in a minimum unit of mt-nucleoid. In contrast, cells that were grown anaerobically in the same medium had giant mt-nucleoids in which, on average, $20.3 \mathrm{mtDNA}$ molecules were involved per mt-nucleoid. Because the large aggregates of mt-nucleoids in anaerobically-grown cells resemble the aggregated form of mt-nucleoids in an mgt 1 mutant strain which lacked a cruciform cutting endonuclease (Lockshon et al. 1995), it is possible that mtDNA molecules are linked by intermediate recombination junctions in cells that reach to the stationary phase in anaerobic conditions. Whereas the size and number of mt-nucleoids distinctly changed between aerobically-grown cells and anaerobicallygrown cells, the total mtDNA content of the cells did not vary markedly. The decrease in total mtDNA content in anaerobically-grown cells that exhibited a very low level of respiration activity was only $27 \%$ compared to the total mtDNA content of aerobically-grown cells. Fukuhara (1969) obtained a similar result in biochemical analyses of the mtDNA content in anaerobically-grown and aerobically-grown cells. In $S$. cerevisiae, the level of respiration activity seems to be correlated to the morphological changes of mt-nucleoids (dispersion into small mt-nucleoids or massive aggregation of the mt-nucleoids) without significant changes in total mtDNA content in cells.

The present results pose a question about how the size and number of mt-nucleoids in a cell are regulated. Recently, MacApline et al. (2000) have reported that a pathway for a general amino 
acid control regulates the number of mt-nucleoids in a cell via the positive transcriptional regulator, Gcn4p. According to this hypothesis, when cells are starved for amino acids, some amino acid biosynthetic pathways are activated, which in turn results in an increase in the number of mt-nucleoids. Conversely, the addition of casamino acids to the minimal medium leads to a decrease in the number of mt-nucleoids in cells.

We noticed a difference in the number of mt-nucleoids in cells between the two aerobic cultures (BT medium and AN medium). At present, we cannot exactly compare the nutrient compounds involved in each medium, because so many different nutrient compounds are involved. Nevertheless, it seems likely that the AN medium contained many more amino acids than the BT medium, because the AN medium was enriched with $0.5 \%$ casamino acids and $1 \%$ yeast extract. Contrary to the hypothesis of MacApline et al. (2000), the number of mt-nucleoids in cells grown in the AN medium was much more than 2-fold that grown in the BT medium. This result seems to suggest that some nutrient compounds beside amino acids may also play roles in regulating the number of mt-nucleoids. We are continuing to investigate the compounds that affected the number and distribution of the mt-nucleoids of yeast cells.

In our estimation, the percentage of mtDNA in total cell DNA exceeded $30 \%$ (aerobic culture in BT medium, 40.3\%; aerobic culture in AN medium 37.2\%, anaerobic culture in AN medium $30.0 \%$ ), when the genome length of the $S$. cerevisiae haploid cell was calculated as $13,389 \mathrm{kbp}$ (Goffeau et al. 1996). These values seem to be much higher than those (5-25\%) widely accepted (Dujon 1981). The reason for this may be the difference in strain used, because the strain used in this study seemed to have far more mt-nucleoids than other laboratory strains, as revealed by DAPIstaining.

\section{Acknowledgments}

This work was supported in part by the NIBB and by a Grant-in-Aid (No. 61540488) for Scientific Research from the Ministry of Education, Science and Culture of Japan.

\section{References}

Dujon, B. 1981. Mitochondrial genetics and functions. In: Strathern, J. N., Jones, E. W. and Broach, J. R. (eds.). The Molecular Biology of The Yeast Saccharomyces. Life Cycle and Inheritance. Cold Spring Harbor Laboratory Press, Cold Spring Harbor, NY. pp. 505-635.

Fukuhara, H. 1969. Relative proportions of mitochondria and nuclear DNA in yeast under various conditions of growth. Eur. J. Biochem. 11: 135-139.

Goffeau, A., Barrell, B. G., Bussey, H., Davis, R. W., Dujon, B., Feldmann, H., Galibert, F., Hoheisel, J. D., Jacq, C., Johnston, M., Louis, E. J., Mewes, H. W., Murakami, Y., Philippsen, P., Tettelin, H. and Oliver, S. G. 1996. Life with 6000 genes. Science 274: 546-567.

Kawano, S., Takano, H. and Kuroiwa, T. 1995. Sexuality of mitochondria: Fusion, recombination, and plasmids. Int. Rev. Cytol. 161: 50-110.

Kuroiwa, T. 1982. Mitochondrial nuclei. Int. Rev. Cytol. 75: 1-59.

Lockshon, D., Zweifel, S. G., Freeman-Cook, L. L., Lorimer, H. E., Brewer, B. J. and Fangman, W. L. 1995. A role for recombination junctions in the segregation of mitochondrial DNA in yeast. Cell 81: 947-955.

MacApline, D. M., Perlman, P. S. and Butow, R. A. 2000. The number of individual mitochondrial DNA molecules and mitochondrial DNA nucleoids in yeast are co-regulated by the general amino acid control pathway. The EMBO Journal 19: 767-775.

Miyakawa, I., Aoi, H., Sando, N. and Kuroiwa, T. 1984. Fluorescence microscopic studies of mitochondrial nucleoids during meiosis and sporulation in the yeast, Saccharomyces cerevisiae. J. Cell Sci. 66: 21-38.

-, Fumoto, S., Kuroiwa, T. and Sando, N. 1995. Characterization of DNA-binding proteins involved in the assembly of mitochondrial nucleoids in the yeast Saccharomyces cerevisiae. Plant Cell Physiol. 36: 1179-1188.

—, Higo, K., Osaki, F. and Sando, N. 1994. Double staining of mitochondria and mitochondrial nucleoids in the living yeast during the life cycle. J. Gen. Appl. Microbiol. 40: 1-14. 
—, Kitamura, Y., Jyozaki, T., Sato, H. and Umezaki, T. 2000. Simple detection of a yeast mitochondrial DNA-binding protein, Abf2p, on SDS-DNA gels. J. Gen. Appl. Microbiol. 46: 311-316.

—, Okazaki-Higashi, C., Higashi, T., Furutani, Y. and Sando, N. 1996. Isolation and characterization of mitochondrial nucleoids from the yeast Pichia jadinii. Plant Cell Physiol. 37: 816-824.

—, Sando, N., Kawano, S., Nakamura, S. and Kuroiwa, T. 1987. Isolation of morphologically intact mitochondrial nucleoids from the yeast, Saccharomyces cerevisiae. J. Cell Sci. 88: 431-439.

- and Sato, H. 2001. Analysis of mitochondrial nucleoid proteins of yeast Saccharomyces cerevisiae by two dimensional gel electrophoresis. Cytologia 66: 99-104.

Newman, S. M., Zelenaya-Troitskaya, O., Perlman, P. S. and Butow, R. A. 1996. Analysis of mitochondrial DNA nucleoids in wild-type and a mutant strain of Saccharomyces cerevisiae that lacks the mitochondrial HMG-box protein, Abf2p. Nucleic Acid Res. 24: 386-393.

Piskur, J. 1994. Inheritance of the yeast mitochondrial genome. Plasmid 31: 229-241.

Sando, N. and Miyake, S. 1971. Biochemical changes in yeast during sporulation. I. Fate of nucleic acids and related compounds. Dev. Growth Differ. 12: 273-283.

Sato, H., Tachifuji, A., Tamura, M. and Miyakawa, I. 2002. Identification of the YMN-1 antigen protein and biochemical analyses of protein components in the mitochondrial nucleoid fraction of the yeast Saccharomyces cerevisiae. Protoplasma 219: 51-58.

Shiiba, D., Fumoto, S.-I., Miyakawa, I. and Sando, N. 1997. Isolation of gaint mitochondrial nucleoids from the yeast Saccharomyces cerevisiae. Protoplasma 198: 177-185.

Tustanoff, E. R. and Bartley, W. 1964. Development of respiration in yeast grown anaerobically on different carbon sources. Biochem. J. 91: 595-600.

Williamson, D. H., Johnston, L. H., Richmond, M. V. and Game, J. C. 1977. Mitochondiral DNA and the heritable unit of the yeast mitochondrial genome: a review. In: Bandlow, W., Schweyen, R. J., Wolf, K. and Kaudewitz, F. (eds.). Mitochondria 1977. Genetics and Biogenesis of Mitochondria. Walter de Gruyter, Berlin. pp. 1-24. 